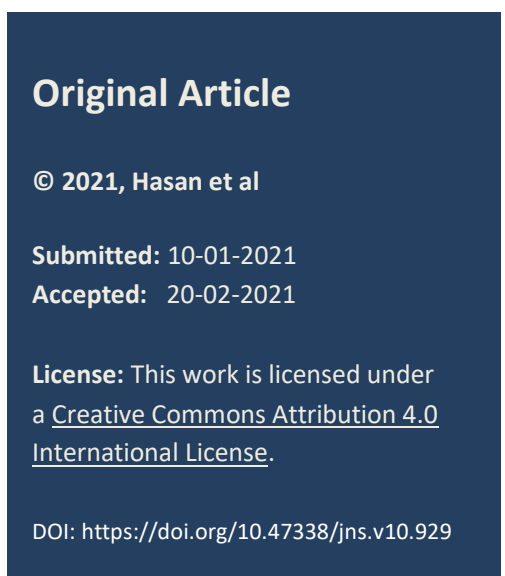

\title{
Tongue shaped oropharyngeal teratoma with cleft palate in a neonate: A case report
}

Samiul Hasan,,$^{*}$ Nadia Afroz, ${ }^{2}$ Jiaul Reza, $^{3}$

1 Division of Pediatric Surgery, Dhaka Shishu (Children) Hospital, Dhaka, Bangladesh

2 National Institute of Mental Health, Dhaka, Bangladesh

3 Department of Pediatric Surgery, Dhaka Medical College Hospital, Dhaka, Bangladesh

Correspondence*: Dr. Md. Samiul Hasan. Assistant Professor, Division of Pediatric Surgery, Dhaka Shishu (Children) Hospital, Dhaka. E-mail: samiulo45@gmail.com

\section{KEYWORDS \\ Oropharyngeal teratoma, \\ Cleft palate, \\ Neonatal tumor, \\ Epignathus}

\begin{abstract}
Background: Atypical presentation of an atypical abnormality makes the diagnosis challenging. Oropharyngeal teratoma (epignathus) is a rare, potentially life-threatening neonatal tumor. Its atypical presentation may cause a delay in diagnosis and increase morbidity and mortality.
\end{abstract}

Case presentation: A newborn girl with oropharyngeal teratoma and cleft palate presented with feeding difficulty. The airway was patent. The tumor was tongue-shaped, smoothwalled, displacing the native tongue, and prevented the fusion of two palatine halves resulting in cleft palate. On the 4th day of life, complete excision was done transorally. Histopathology revealed a mature teratoma. There was no sign of recurrence at three months of follow-up.

Conclusion: Presentation of the oropharyngeal teratoma varies according to its site, size, and extension. Timely diagnosis is essential to avoid life-threatening respiratory obstruction. Complete excision of the mass is usually associated with a good prognosis.

\section{INTRODUCTION}

Oropharyngeal tumors in neonatal age are rare and include lymphangioma, glioma, encephalocele, hemangioma, and teratoma. The tumor's position and size determine the outcome in most cases rather than the tumor's nature.[1] A mature oropharyngeal teratoma, also known as epignathus, is a rare congenital tumor affecting only 1 in 35,000-200,000 live births. Like teratoma in other parts of the body, epignathus shows a female predominance.[2,3] Only $6 \%$ of these tumors are associated with palate and tongue malformation. $[2,4]$

Timely diagnosis and appropriate management are critical for avoiding complications like life-threatening respiratory obstruction, feeding difficulties, and recurrent aspiration pneumonia.[5,6] Though postnatal diagnosis is evident in most cases because of the oral cavity's protruded mass, small masses at a problematic location can lead to diagnostic difficulty.[6]

We present a case of oropharyngeal teratoma presenting as a tongue-shaped mass that was initially misdiagnosed as macroglossia.

\section{CASE REPORT}

A 2-day-old female neonate was referred from the neonatology department to evaluate feeding difficulty with macroglossia suspicion. The antenatal period was uneventful except for mild polyhydramnios. The baby was the 1 st order birth of a 25 years old healthy mother, delivered vaginally at term with an APGAR score of 10 at 5 minutes, and the birth weight was 3.2 $\mathrm{kg}$. During the first breastfeeding trial, the mother noticed that the baby could not suck properly, and the tongue is larger than usual.

At presentation, the baby was pink, with stable vitals and normal reflexes. On examination of the oral cavity, a tongue-shaped mass was originating from the palate's left half protruded forwards. It displaced the native tongue downwards, which was hypoplastic due to the tumor's pressure effect (Fig.1). The mass was firm, covered with skin containing hair follicles (Fig.1). After moving the mass downwards and outwards, cleft was visible on the right side. No other gross congenital anomaly was found.

Based on the working diagnosis of an epignathus, we checked the serum alpha-fetoprotein level, which was 
within the normal limits $(9700 \mathrm{ng} / \mathrm{ml})$ for age. The decision for emergency excision of the mass without further imaging study was taken because of the risk of respiratory obstruction.

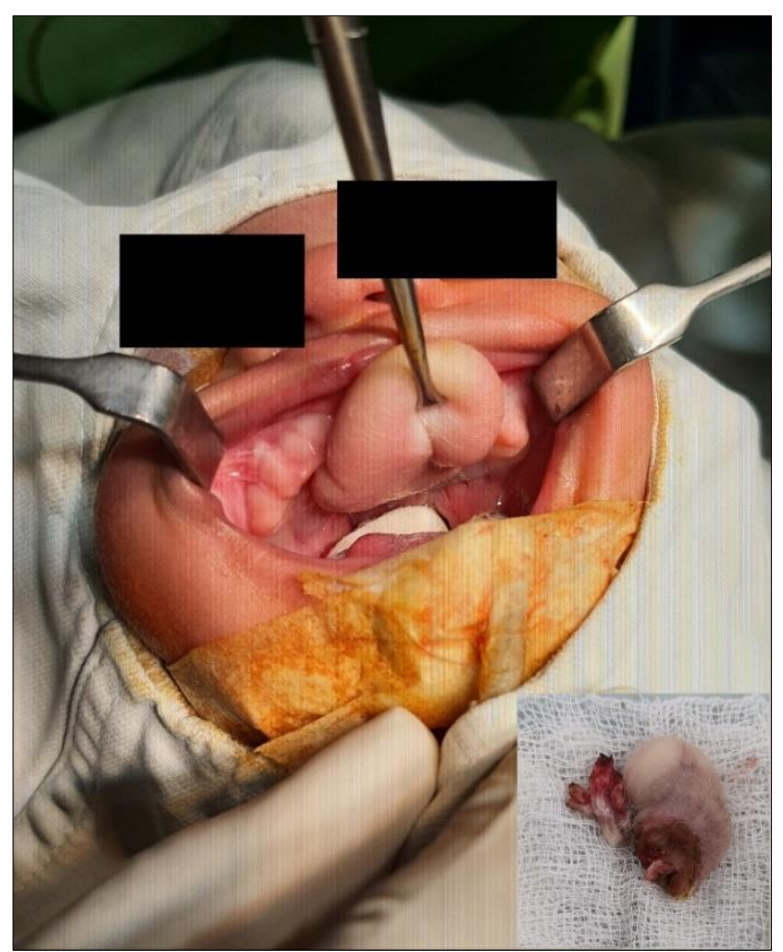

Figure 1: Tongue-shaped growth arising from the palate. Inset showing excised tissue.

Intubation was possible after pulling the mass upward. Complete excision of the mass was possible transorally using monopolar diathermy. After excision, the palate's mucosal wound was left open for secondary healing. The postoperative period was uneventful. The repair of the palate shall be planned after nine months of age.

Histopathology report showed mature teratoma. Clinical follow-up at three months showed no sign of recurrence.

\section{DISCUSSION}

Though teratomas are the most typical congenital tumor, their oral cavity occurrence, especially in the palate, is infrequent. Only 24 case reports are available in English medical literature.[1,4,5] Cleft palate in these cases results from mechanical obstruction preventing the fusion of palatine shelves.[7] The lateral displacement of the cleft in our case also supports this theory. This mechanical obstruction can also lead to the bifid tongue, bifid nose, or even cardiac and cranial anomalies during the development.[7]

The etiopathogenesis of teratoma is still not fully established. Abnormal proliferation of the sequestered pluripotent stem cell is the most accepted hypothesis of teratoma.[5] Some studies found an association of chromosomal anomalies like duplication of $1 \mathrm{q}$ and 19p, X chromosome mosaicism, and 49 XXXXY karyotype with epignathus, while other studies denied chromosomal abnormalities. Any environmental risk factor in the etiopathogenesis of epignathus is not found yet.[1,2] We did not perform a chromosomal analysis of our patient.

In most cases, diagnosis is possible on clinical examination showing an irregular mass of different sizes arising from the oral cavity. Intracranial extension of the mass has also been reported.[2] Prenatal diagnosis was possible in 14 out of 24 reported cases of epignathus. Prenatal ultrasonography can detect oropharyngeal teratoma with the advancement of the pregnancy as the tumor grows with time. It requires special attention during the evaluation of the differential diagnosis of polyhydramnios. Magnetic resonance imaging (MRI) can better delineate the tumor's extension and guide the management plan. MRI could be helpful in both prenatal and postnatal settings.[8-10] The regular shape of the tumor in our patient resembled the tongue when the original tongue was hypoplastic, which caused the initial misdiagnosis. As we did not have access to in-house MRI, we planned for surgery without delay to avoid complications.

If the prenatal evaluation reveals a risk of airway obstruction, fetal management is an option when available.[10] After delivery, the most urgent and critical task is to secure the airway. Some reports show the successful outcome of the ex-utero intrapartum (EXIT) procedure and or tracheostomy to maintain the airway.[1,5,8] Fortunately, our patient could maintain the airway because of the relatively small size of the tumor. The treatment of choice is complete primary excision of the tumor earliest possible as it grows with the baby. Transoral excision of the tumor is usually possible, but some cases require additional neck dissection to complete the tumor removal.[5,9]

Oropharyngeal teratomas are usually mature and have an excellent prognosis when excised completely.[5] Four reported cases of immature oral teratoma showed a uniform lethal prognosis.[4] Another lethal prognostic factor is intracranial extension.[2]

In conclusion, oropharyngeal teratomas (epignathus) are rare congenital tumors. The outcome depends on the nature and extent of the tumor. Antenatal diagnosis allows preparation for optimal perinatal management with a favorable outcome.

\section{Acknowledgements: $\mathrm{Nil}$}

Conflict of Interest: Authors have no conflict of interest.

Source of Support: Nil

Consent to Publication: Author(s) declared taking informed written consent for the publication of clinical photographs/material (if any 
used), from the legal guardian of the patient with an understanding that every effort will be made to conceal the identity of the patient, however it cannot be guaranteed.

\section{REFERENCES}

1. Bahgat $\mathrm{M}$, Bahgat $\mathrm{Y}$, Bahgat A. Oropharyngeal teratoma, a rare cause of airway obstruction in neonates. BMJ Case Rep. 2012; 2012:bcr2012006580.

2. Kirishima M, Yamada S, Shinya M, Onishi S, Goto Y, Kitazono I, et al. An autopsy case of epignathus (immature teratoma of the soft palate) with intracranial extension but without brain invasion: case report and literature review. Diagn Pathol. 2018; 13:99.

3. Chauhan DS, Guruprasad Y, Inderchand S. Congenital nasopharyngeal teratoma with a cleft palate: case report and a 7 year follow up. J Maxillofac Oral Surg. 2011; 10:253-6.

4. Kishi Y, Soh S, Tanaka S, Nitta A, Matsushima A. A case of congenital teratoma with cleft palate in a neonate. Arch Clin Med Case Rep. 2020; 4:746-53.

5. Aubin A, Pondaven S, Bakhos D, Lardy H, Robier A, Lescanne E. Oropharyngeal teratomas in newborns: Management and outcome. Eur Ann Otorhinolaryngol Head Neck Dis. 2014; 131:271-5.

6. Posod A, Griesmaier E, Brunner A, Pototschnig C, Trawöger R, Kiechl-Kohlendorfer U. An unusual cause
Author Contributions: Author(s) declared to fulfil authorship criteria as devised by ICMJE and approved the final version.

of inspiratory stridor in the newborn: congenital pharyngeal teratoma--a case report. BMC Pediatr. 2016; $16: 1$.

7. Jie H, Yanan W, Hanguang $Z$, Weiliu Q, Yue $H$. Nasopharyngeal teratoma associated with cleft palate in newborn: report of 2 cases. Oral Surg Oral Med Oral Pathol Oral Radiol Endod. 2010; 109:211-6.

8. Morof D, Levine D, Grable I, Barnewolt C, Estroff J, Fishman S, et al. Oropharyngeal teratoma: prenatal diagnosis and assessment using sonography, MRI, and CT with management by ex utero intrapartum treatment procedure. Am J Roentgenol. 2004; 183:4936.

9. Manchali MM, Sharabu C, Latha M, Kumar L. A rare case of oropharyngeal teratoma diagnosed antenatally with MRI. J Clin Imaging Sci. 2014; 4:15.

10. Kontopoulos EV, Gualtieri M, Quintero RA. Successful in utero treatment of an oral teratoma via operative fetoscopy: case report and review of the literature. Am J Obstet Gynecol. 2012; 207:e12-5. 